\title{
Long noncoding RNA HULC accelerates the growth of human liver cancer stem cells by upregulating CyclinD1 through miR675- PKM2 pathway via autophagy
}

Chen Wang ${ }^{\dagger}$, Xiaoxue Jiang ${ }^{\dagger}$, Xiaonan Li ${ }^{\dagger}$, Shuting Song, Qiuyu Meng, Liyan Wang, Yanan Lu, Xiaoru Xin, Hu Pu, Xin Gui, Tianming Li and Dongdong Lu*

\begin{abstract}
Background: The functions of HULC have been demonstrated in several cancers. However, its mechanism has not been elucidated in human liver cancer stem cells.

Methods: Liver cancer stem cells were isolated from Huh7 cells; gene infection and tumorigenesis test in vitro and in vivo were performed.

Results: We demonstrate that HULC promotes growth of liver cancer stem cells in vitro and in vivo. Mechanistically, HULC enhances the expression of Sirt1 dependent on miR675 and then induces the cellular autophagy through Sirt1. HULC enhances CyclinD1 and thereby increases pRB and inhibited P21 WAF1/CIP 1 via autophagy-miR675-PKM2 pathway in human liver cancer stem cells. Ultimately, our results demonstrate that CyclinD1 is required for the oncogenic functions of HULC in liver cancer stem cells.
\end{abstract}

Conclusions: It reveals the key molecular signaling pathways for HULC and provides important basic information for finding effective tumor therapeutic targets based on HULC.

Keywords: HULC, CyclinD1, miR675, PKM2, Autophagy

\section{Introduction}

HULC has been studied in several cancers and promotes tumorigenesis [1-4]. Furthermore, HULC polymorphisms are associated with hepatocellular cancer risk and prognosis $[5,6]$. In addition, HULC enhances autophagy [7] and facilitates hepatocellular carcinoma genesis [8]. Furthermore, HULC regulates the expression of bone morphogenetic protein9 (BMP9) [9]. Interestingly, HULC acts as an oncogene [10] and inhibits apoptosis [11] and promotes invasion [12, 13]. Furthermore, HULC stabilizes Sirt1 and decreases the chemosensitivity [14]. Moreover, HULC aggravates the cellular proliferation by regulating telomere repeat-binding factor2 [15] and CUDR, $\beta$-Catenin [16], and IGF2 mRNAbinding protein 1 (IGF2BP1) [17].

\footnotetext{
* Correspondence: ludongdong@tongji.edu.cn

${ }^{+}$Chen Wang, Xiaoxue Jiang and Xiaonan Li contributed equally to this work. Shanghai Putuo District People's Hospital, School of Life Science and Technology, Tongji University, Shanghai 200092, China
}

In this study, HULC is associated with miRNA675, Sirt1, CyclinD1, and autophagy. A study indicates that miR-675 enhances cell proliferation [18, 19] and Smads/miR-675/ TGF $\beta$ R1 axis modulates the proliferation [20]. Moreover, sPIF promotes myoblast differentiation via the H19/miR675/let-7 pathways [21] Furthermore, miR-675 mediates therapeutic effect [22]. A study indicates that SIRT1 is implicated in stem cell homeostasis. In particular, Conditional Sirt1 deletion in the hematopoietic stem and progenitor system promotes hematopoietic stem and progenitor cell (HSPC) expansion under stress conditions [23]. Moreover, SIRT1 enhances progression and epithelial-mesenchymal transition in several cancer [24, 25]. Furthermore, CyclinD1 promotes the cancer cell growth dependent on autophagy [26]. A study shows that CyclinD1 complement p16 acts as tumor marker [27] and shows heterogeneous expression of $\mathrm{pRb}$ and CyclinD1 [28]. Importantly, autophagy is essential in cellular processes [29]. For example, downregulation of

(c) The Author(s). 2020 Open Access This article is distributed under the terms of the Creative Commons Attribution 4.0 International License (http://creativecommons.org/licenses/by/4.0/), which permits unrestricted use, distribution, and 
CD44v6 inhibits autophagy in colorectal cancer HT29 cells [30], and LncRNA CCAT1 functions as apoptosis inhibitor via autophagy inhibition [31] and upregulated lysine-specific demethylase 4B by autophagy [32]. Notably, BCR signaling contributes to autophagy regulation [33].

In this study, our observations suggest that HULC promotes progression of liver cancer stem cells dependent on CyclinD1. It provides important basic information for finding effective tumor therapeutic targets.

\section{Materials and methods}

\section{Cell infection and transfection}

Cells were infected with lentivirus and transfected with DNA plasmids according to the manufacturer's instructions (also see Additional file 1).

\section{MicroRNA detection}

Real-time RT-PCR-based detection of mature miR-675 was achieved with the miRNA Detection kit and miR675-specific upstream primers (5'-TGGTGCGGAGAG GGCCCACAGTG-3').

\section{RNA immunoprecipitation (RIP)}

Ribonucleoprotein particle-enriched lysates were incubated with protein A/G-plus agarose beads (Santa Cruz, Biotechnology, Inc.CA) together with the primary antibody or normal IgG for $4 \mathrm{~h}$ at $4{ }^{\circ} \mathrm{C}$. Beads were subsequently washed and RNAs were then isolated. RT-PCR was performed according to the manufacturer's instructions.

\section{Cells proliferation CCK8 assay}

Cells were grown in complete medium for CCK8 assay according to the manufacturer's instructions. Cell growth curve was based on the values of OD450.

\section{Colony-formation efficiency assay}

Cell colonies on the dish were stained with Crystal Violet (Henan Tianfu Chemical Co., Ltd.), and the colonies were counted according to the manufacturer's instructions.

\section{Xenograft transplantation in vivo}

Four-week male athymic Balb/C mice were purchased from Shi Laike Company (Shanghai, China). The athymic Balb/C mice were injected at the armpit area subcutaneously with suspension of cells. The wet weight of each xenograft was determined for each mouse. The use of mice for this work was reviewed and approved by the institutional animal care and use committee in accordance with China National Institutes of Health guidelines.

\section{Results}

HULC promotes growth of liver cancer stem cells

To demonstrate the effect of HULC on human liver cancer stem cells, we perform the tumorigenesis test in vitro. First, human liver cancer stem cells were isolated from Huh7 cells. Cells that meet the four indexes of CD133+, CD44+, CD24+, and EpCAM+ are defined as human liver cancer stem cells (hLCSCs), and cells that satisfy the four indexes of CD133-, CD44-, CD24-, and EpCAM are defined as non-hepatoma stem cells (non-hLCSCs). CD44, CD24, and EpCAM are expressed in hLCSCs, but not in non-hLCSCs (Additional file 1: Figure S1). Moreover, the sphere formation rate is $0.153 \pm 0.0372 \%$ in the hLCSCs group, and the sphere formation rate is 0 in the nonhLCSCs group $(0.153 \pm 0.0372 \%$ vs $0, P=0.00079<0.01)$ (Additional file 1: Figure S2A). The weight of xenograft tumors is $0.68 \pm 0.19 \mathrm{~g}$ in the hLCSCs group, and the weight of xenograft tumors is $0 \mathrm{~g}$ in the non-hLCSCs group (0.68 \pm $0.19 \mathrm{~g}$ vs $0, P=0.00000098<0.01$ ) (Additional file 1 : Figure S2B). Next, we established four stable hLCSC lines transfected with pCMV6-A-GFP (GFP ctrl group), pCMV6-AGFP-HULC (HULC group), pGFP-V-RS (RNAi ctrl group), and pGFP-V-RS-HULC (HULCi group), respectively (Fig. 1a). As shown in Fig. 1b, HULC expression was significantly enhanced in the HULC group compared with the GFP ctrl group and reduced in the HULCi group compared with the RNAi ctrl group. As shown in Fig. 1c, the growth ability was significantly increased in the HULC group compared to the GFP ctrl group $(P<0.01)$ and decreased in the HULCi group compared to the RNAi ctrl group compared to the GFP ctrl group $(P<0.01)$. Moreover, the proportion of BrdU-positive cells in the HULC group was significantly increased in the HULC group compared to the GFP ctrl group $(P<0.01)$ and decreased in the HULCi group compared to the RNAi ctrl group compared to GFP the ctrl group $(P<0.01)$ (Fig. 1d). Furthermore, the soft-agar colony formation rate was significantly increased in the HULC group compared to the GFP ctrl group $(31.09 \pm 7.29 \%$ vs $65.30 \pm 11.58 \%, P=0.0053<0.01)$ and decreased in the HULCi group compared with the RNAi ctrl group $(34.049 \pm 4.79 \%$ vs $17.34 \pm 1.37 \%, P=0.0102<0.05)$ (Fig. 1e). The sphere-formation rate of hLCSCs was significantly increased in the HULC group compared to the GFP ctrl group $(28.74 \pm 6.47 \%$ vs $54.71 \pm 8.19 \%$, $P=0.0008<0.01)$ and decreased in the HULCi group compared with the RNAi ctrl group $(24.52 \pm 4.31$ vs $10.03 \pm 2.67 \%, P=0.031<0.05$ ) (Fig. 1f). Collectively, these results suggest that HULC promotes the growth in vitro of liver cancer stem cells.

\section{HULC accelerates growth of hLCSCs in vivo}

To investigate the effect of HULC on hLCSCs in vivo, the four stable hLCSC lines were injected subcutaneously into Balb/C mice, respectively. As shown in Fig. 2a and b, compared with the GFP ctrl group, the weight of xenograft tumor was increased approximately by twofold in the HULC group $(0.385 \pm 0.057 \mathrm{~g}$ vs $0.852 \pm 0.108 \mathrm{~g}, p=0.000007933<$ 0.01); however, compared with the RNAi ctrl group, the 


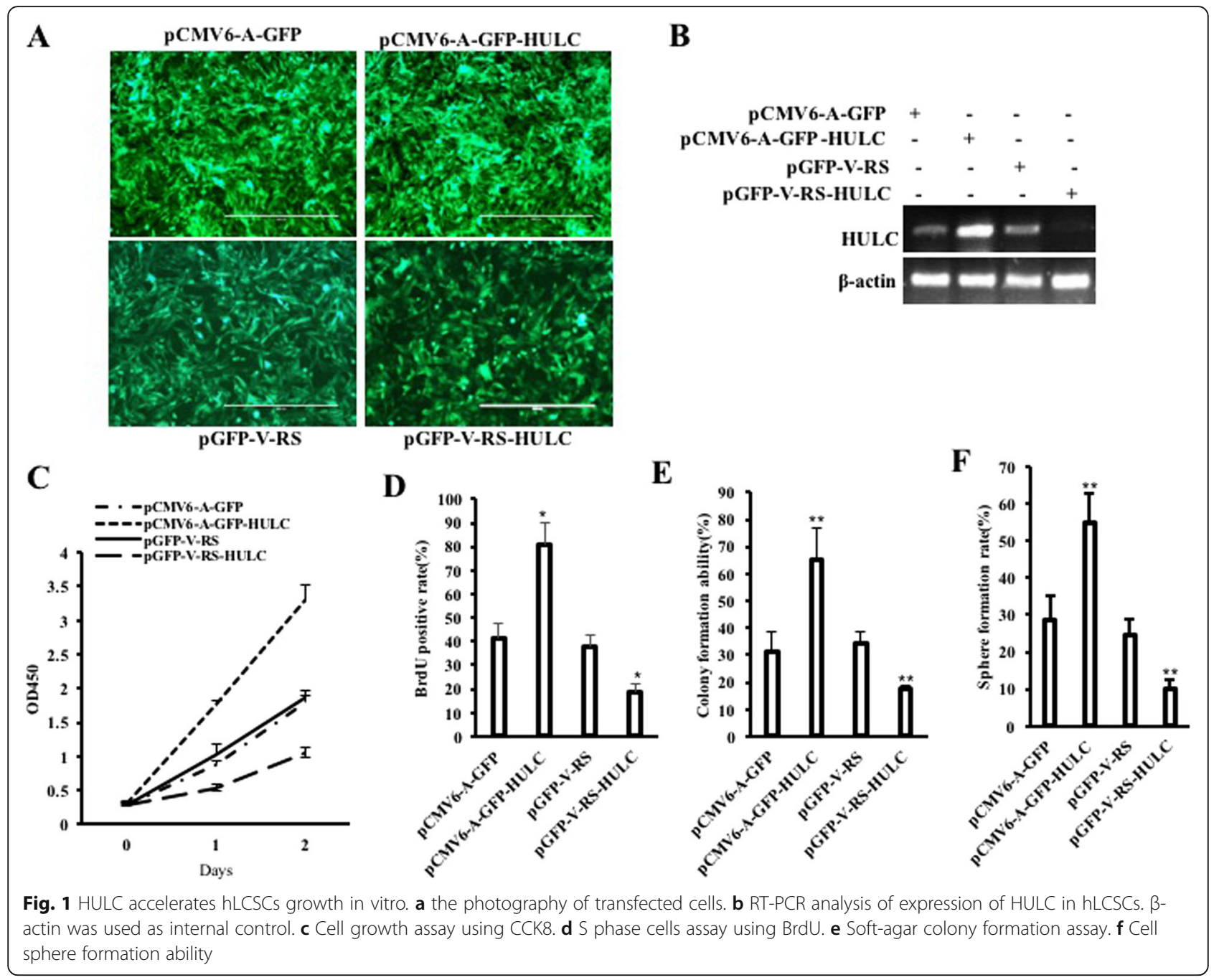

weight of xenograft tumor was decreased approximately by one third in the HULCi group $(0.45 \pm 0.068$ $\mathrm{g}$ vs $0.153 \pm 0.0372 \mathrm{~g}, p=0.000224907<0.01)$. Furthermore, the xenograft tumors appeared earlier in the HULC group than in the GFP ctrl group $(8.33 \pm 1.37$ days vs $5.67 \pm 0.816$ days, $p=0.0014788<0.01$ ), whereas those appeared later in the HULCi group than in the RNAi ctrl group $(9.0 \pm 1.79$ days vs $15.5 \pm 2.43$ days, $p=0.000184<0.01$ ) (Fig. 2c). Furthermore, xenograft tumor differentiation was poorer in the HULC group than in the GFP ctrl group, whereas xenograft tumor differentiation was well in the HULCi group than in the RNAi ctrl group (Fig. 2d). Furthermore, the PCNA-positive rate was significantly higher in the HULC group than in the GFP ctrl group $(36.15 \pm 7.25 \%$ vs $69.99 \pm 8.24 \%, p=0.00041<0.01)$ and lower in the HULCi group than in the RNAi ctrl group $(34.62 \pm 4.94 \%$ vs $18.19 \pm 2.67 \%, p=0.00029<0.01$ ) (Fig. $2 \mathrm{~d}, \mathrm{e}$ ). Together, these results suggest that HULC accelerates growth of liver cancer stem cells in vivo.
HULC increases the miR675 in liver cancer stem cells Given that HULC promotes the growth of liver cancer stem cells and miR675 is associated with oncogenesis, we consider whether HULC regulates the expression of miR675. To address this hypothesis, we measured the level of RNA methylation of pri-miR675 in hLCSCs. Our results showed that excessive HULC increases and HULC knockdown decreased the binding of METTL3 (a RNA methyltransferase) to pri-miR675 compared to the control group (Fig. 3a). Furthermore, real-time RIP results showed that the binding of METTL3 (a RNA methyltransferase) to pri-miR675 was increased in the pCMV6-A-GFP-HULC group compared to the pCMV6A-GFP group and decreased in the pGFP-V-RS-HULC group compared to the pGFP-V-RS group (Fig. $3 b$ ). In particular, pri-miR675, pre-miR675, and mature miRR675 were significantly increased in the PCMV6-A-GFPHULC group compared to the pCMV6-A-GFP group and decreased in the pGFP-V-RS-HULC group compared to the pGFP-V-RS group (Fig. 3c). Furthermore, 


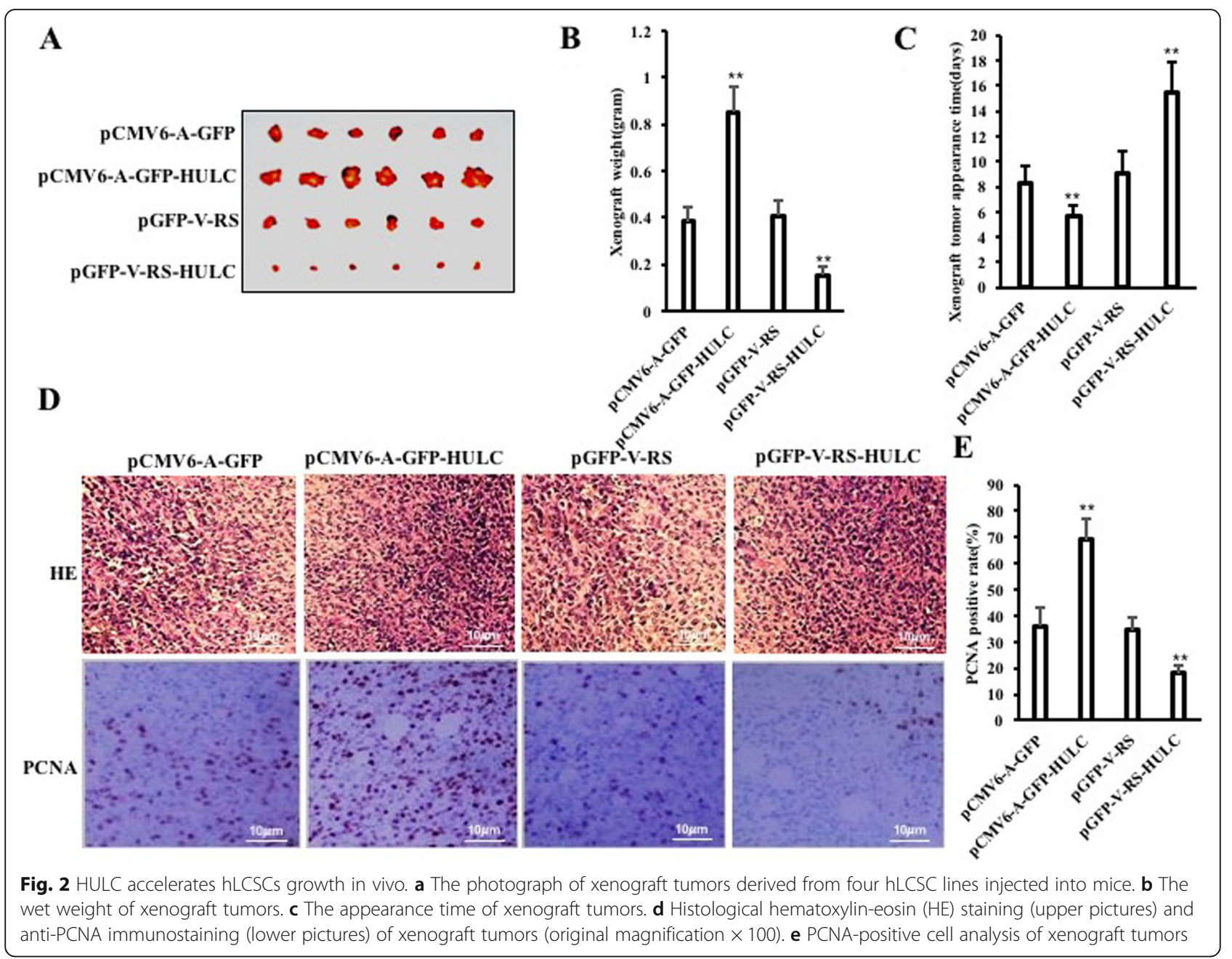

mature miR675 was increased in the pCMV6-A-GFPHULC group compared to the pCMV6-A-GFP group and decreased in the pGFP-V-RS-HULC group compared to the pGFP-V-RS group (Fig. 3d). Although pre-miR675, premiR675, and mature mi-R675 were significantly increased in the pCMV6-A-GFP-HULC group compared to the pCMV6-A-GFP group, it was significantly not altered in the pCMV6-A-GFP-HULC+pGFP-V-RS-METTL3 group compared to the pCMV6-A-GFP group (Additional file 1: Figure S3A\&B). Collectively, these observations suggest that HULC enhances the expression and maturity of miR675 dependent on METTL3.

\section{HULC promotes the expression of Sirt1 dependent on miR675}

Given that HULC increases the expression of miR675, we will try to consider whether HULC regulates expression of Sirt1 via miR675. As shown in Fig. 4a, mature miR675 matches 3' untranslational region (UTR) on histone deacetylase 5(HDAC5) mRNA via eight-seed sequence. Next, as shown in Fig. 4b, although the DHAC5 3'
UTR luciferase activity was significantly reduced in the rLVmiR675 group compared to the rLV control group (199, $362.03 \pm 32,442.268$ vs $12,057.69 \pm 4192.57, p=0.00375<$ 0.01 ), it was significantly not altered in the rLV-miR675 group compared to the rLV group $(118,226.40 \pm 14,210.88$ vs $105,230.04 \pm 22,650.11, p=0.22704>0.05$ ) (Additional file 1: Figure S4). Although the DHAC5 mRNA was not significantly altered between the rLV-miR675 group and rLV control group, the expression of DHAC5 was significantly reduced in the rLV-miR675 group compared to the rLV control group (Fig. 4c). Although the expression of DHAC5 was significantly reduced in the pCMV6-A-GFP-HULC group and increased in the rLV-Cas9-miR675 group compared to the pCMV6-A-GFP group, it was significantly not altered in the pCMV6-A-GFP-HULC plus rLV-Cas9-miR675 group compared to the pCMV6-A-GFP group (Fig. 4d). In particular, the loading of DHAC5 on the Sirt1 promoter region was significantly reduced in the pCMV6-A-GFP-HULC group and increased in the rLV-Cas9-miR675 group compared to the pCMV6-A-GFP group. However, the expression of DHAC5 was significantly not altered in the pCMV6-A-GFP- 


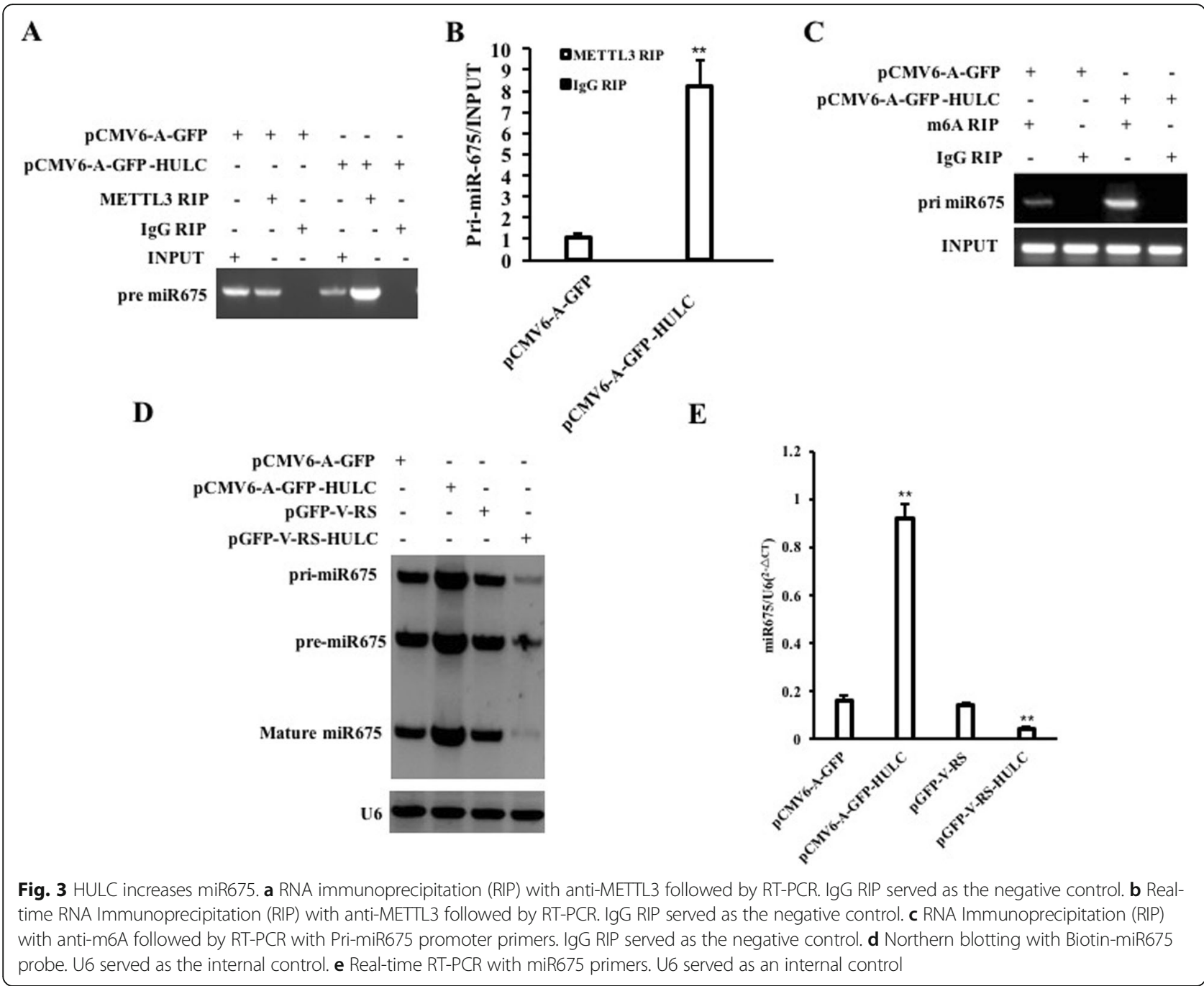

HULC plus rLV-Cas9-miR675 group compared to the pCMV6-A-GFP group (Fig. 4e). Furthermore, although the luciferase activity of Sirt1 promoter was significantly increased in the pCMV6-A-GFP-HULC group $(87,825.04 \pm 10$, 954.98 vs $306,040.71 \pm 27,824.28, \quad p=0.0042<0.01)$ and reduced in the rLV-Cas9-miR675 group compared to the pGFP-V-RS group compared to the pCMV6-A-GFP group $(87,825.04 \pm 10,954.98$ vs $28,809.006 \pm 999.09, p=0.0053<$ 0.01 ), it was significantly not altered in the pCMV6-A-GFPHULC plus rLV-Cas9-miR675 group compared to the pCMV6-A-GFP group $(87,825.04 \pm 10,954.98$ vs 82 , $539.303 \pm 16,170.81, p=0.3625>0.05)$ (Fig. 4f). Moreover, the luciferase activity of Sirt1 promoter was significantly increased in the pCMV6-A-GFP-HULC group compared to the pCMV6-A-GFP group $(10,032.48 \pm 1131.55$ vs 86 , $393.99 \pm 10,824.39, p=0.003835<0.01)$ and reduced in the pGFP-V-RS-HULC group compared to the pGFP-VRS group (9143.86 \pm 1613.94 vs $2522.18 \pm 429.04, p=$ $0.0059436<0.01$ ) (Fig. 4g). Ultimately, the expression of Sirt1 was significantly increased in the pCMV6-A-
GFP-HULC group and decreased in the rLV-Cas9-miR675 group compared to the pCMV6-A-GFP group. However, the expression of Sirt1 was significantly not altered in the pCMV6-A-GFP-HULC plus rLV-Cas9-miR675 group compared to the pCMV6-A-GFP group (Fig. 4h, i). Moreover, although the expression of Sirt1 was significantly increased in the pCMV6-A-GFP-HULC group and decreased in the rLV-Cas9-miR675 group compared to the pCMV6-A-GFP group, it was significantly not altered in the pCMV6-AGFP-HULC plus rLV-HDAC5 group compared to the pCMV6-A-GFP group (Fig. 4j). Collectively, these observations indicate that HULC enhances the expression of Sirt1 dependent on miR675-HDAC5.

\section{HULC increases the autophagy through Sirt1}

Given that HULC increases the expression of Sirt1 which is associated with deacetylation of LC3, we consider whether HULC influences on the autophagy through Sirt1 in liver cancer stem cells. First, although the interaction between Sirt1 and LC3 was significantly increased in the pCMV6-A- 


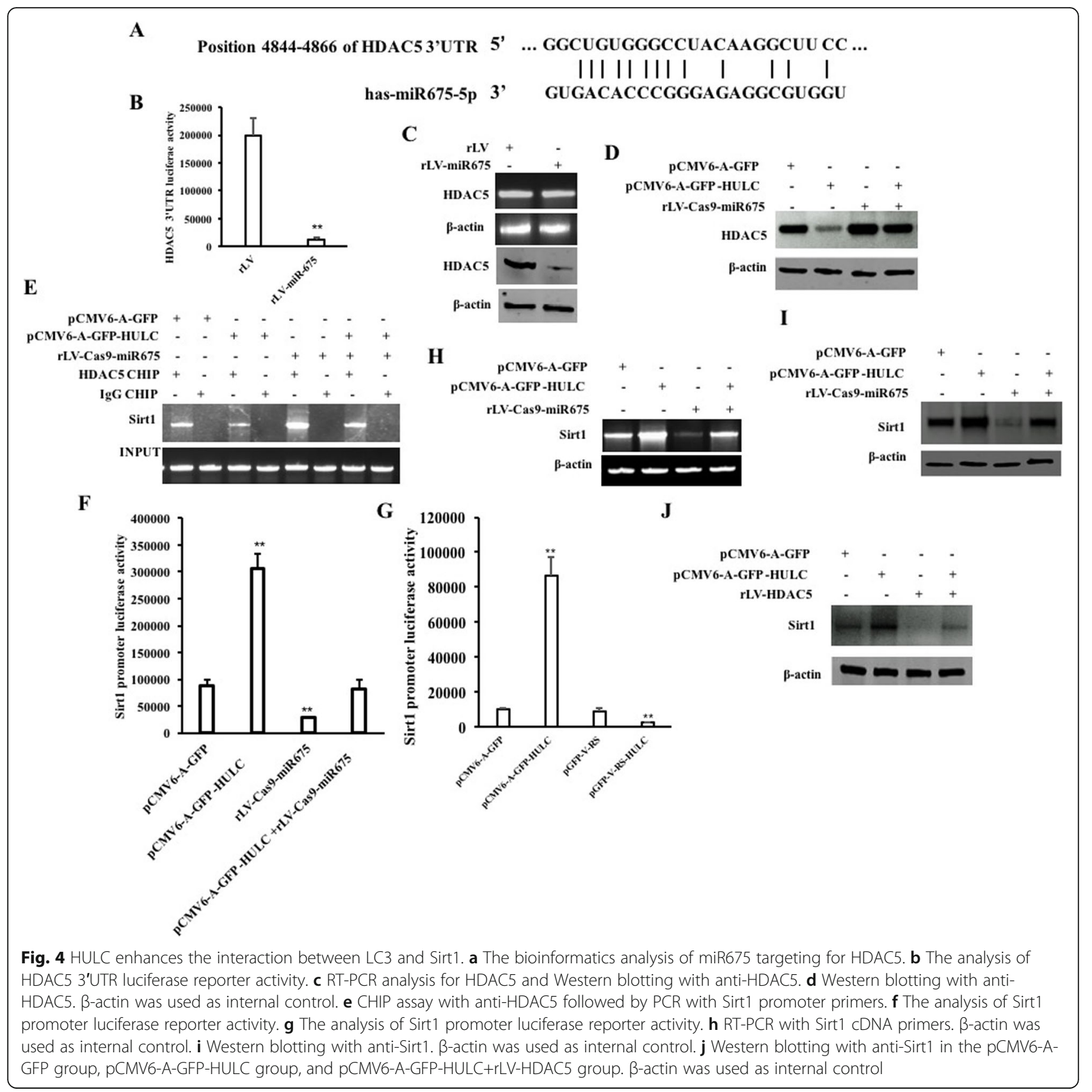

GFP-HULC group and decreased in the rLV-Cas9-miR675 group compared to the pCMV6-A-GFP group, it was significantly not altered in the pCMV6-A-GFP-HULC plus rLV-Cas9-miR675 group compared to the pCMV6-A-GFP group (Fig. 5a). As shown in Fig. 5b, the Ac-LC3 was significantly decreased in the pCMV6-A-GFP-HULC group compared to the pCMV6-A-GFP group. However, the AcLC3 was not significantly altered in the pCMV6-A-GFPHULC plus Sirtinol (a Sirt1 inhibitor) group and the pCMV6-A-GFP-HULC plus rLV-Cas9-miR675 group compared to pCMV6-A-GFP group, respectively. Therefore, although the interaction between LC3 and DOR was significantly increased in the pCMV6-A-GFP-HULC group compared to the pCMV6-A-GFP group, it was not significantly altered in the pCMV6-A-GFP-HULC plus Sirtinol group and the pCMV6-A-GFP-HULC plus rLV-Cas9miR675 group compared to the pCMV6-A-GFP group (Fig. 5c). Strikingly, the interaction between LC3 and ATG4 was significantly increased in the pCMV6-A-GFPHULC group compared to the pCMV6-A-GFP group. However, the interaction between LC3 and DOR was not significantly altered in the pCMV6-A-GFP-HULC plus Sirtinol group and the pCMV6-A-GFP-HULC plus rLVCas9-miR675 group compared to the pCMV6-A-GFP 


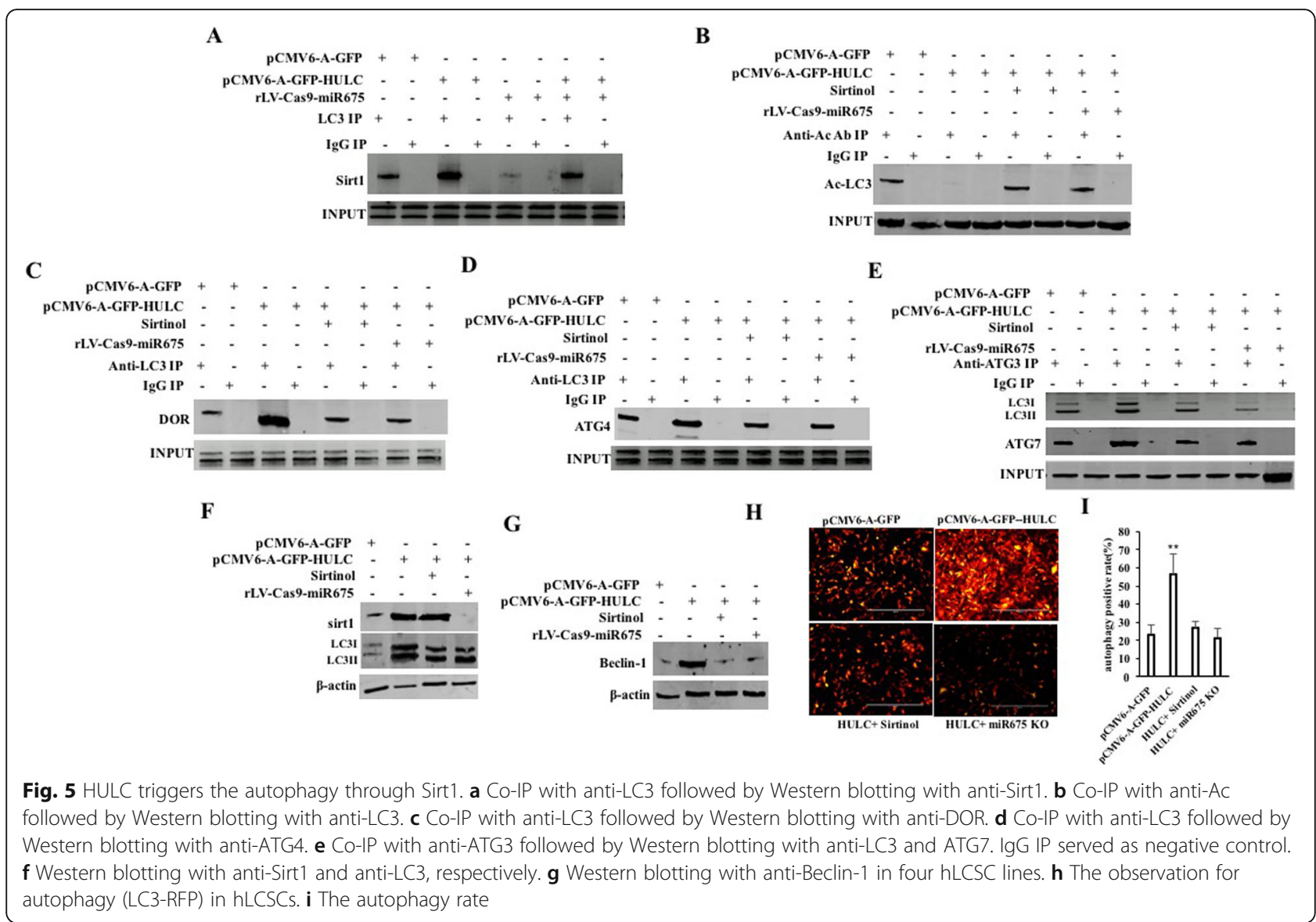

group, respectively (Fig. 5d). Furthermore, the interaction between LC3 and ATG3 was significantly increased in the pCMV6-A-GFP-HULC group compared to the pCMV6A-GFP group. However, the interaction between LC3 and ATG3 was not significantly altered in the pCMV6-AGFP-HULC plus Sirtinol group and the pCMV6-A-GFPHULC plus rLV-Cas9-miR675 group and compared to the pCMV6-A-GFP group, respectively (Fig. 5e). And, the interaction between ATG3 and ATG7 was significantly increased in the pCMV6-A-GFP-HULC group compared to the pCMV6-A-GFP group. However, the interaction between ATG3 and ATG7 was not significantly altered in the pCMV6-A-GFP-HULC plus Sirtinol group and the pCMV6-A-GFP-HULC plus rLV-Cas9-miR675 group compared to the pCMV6-A-GFP group, respectively (Fig. 5e). Thus, the activated LC3II was significantly enhanced in the pCMV6-A-GFP-HULC group compared to the pCMV6-A-GFP group. However, the activated LC3II was not significantly altered in the pCMV6-A-GFPHULC plus Sirtinol group and the pCMV6-A-GFP-HULC plus rLV-Cas9-miR675 group compared to the pCMV6-AGFP group, respectively (Fig. $5 f$ ). In particular, the beclin1 was significantly increased in the pCMV6-A-GFP-HULC group compared to the pCMV6-A-GFP group. However, the beclin1 was not significantly altered in the pCMV6-AGFP-HULC plus Sirtinol group and the pCMV6-A-GFPHULC plus rLV-Cas9-miR675 group compared to the pCMV6-A-GFP group, respectively (Fig. 5g). Ultimately, the autophagy was significantly enhanced in the pCMV6A-GFP-HULC group compared to the pCMV6-A-GFP group $(23.38 \pm 5.27 \%$ vs $56.41 \pm 11.38 \%, p=0.00902<$ $0.01)$. However, the autophagy was not significantly altered in the pCMV6-A-GFP-HULC plus Sirtinol group and the pCMV6-A-GFP-HULC plus rLV-Cas9-miR675 group compared to the pCMV6-A-GFP group, respectively $(23.38 \pm 5.27 \%$ vs $26.93 \pm 3.56 \%, p=0.10548>0.05 ; 23.38 \pm$ $5.27 \%$ vs $21.38 \pm 5.41 \%, p=0.361978>0.05$ ) (Fig. 5 h, i). Collectively, these observations suggest that HULC increases the autophagy dependent on Sirt1.

\section{HULC enhances CyclinD1 to increase pRB and inhibit P21 WAF1/CIP 1 via autophagy-PKM2 pathway}

To address whether HULC influences on the PKM2 in liver cancer stem cells by autophagy, we first analyze the interaction between LC3II and Pyruvate Kinase M2 (PKM2) in liver cancer stem cells. As shown in Fig. 6a, the interaction between LC3II and PKM2 was significantly enhanced in the pCMV6-A-GFP-HULC group compared to the pCMV6-A- 


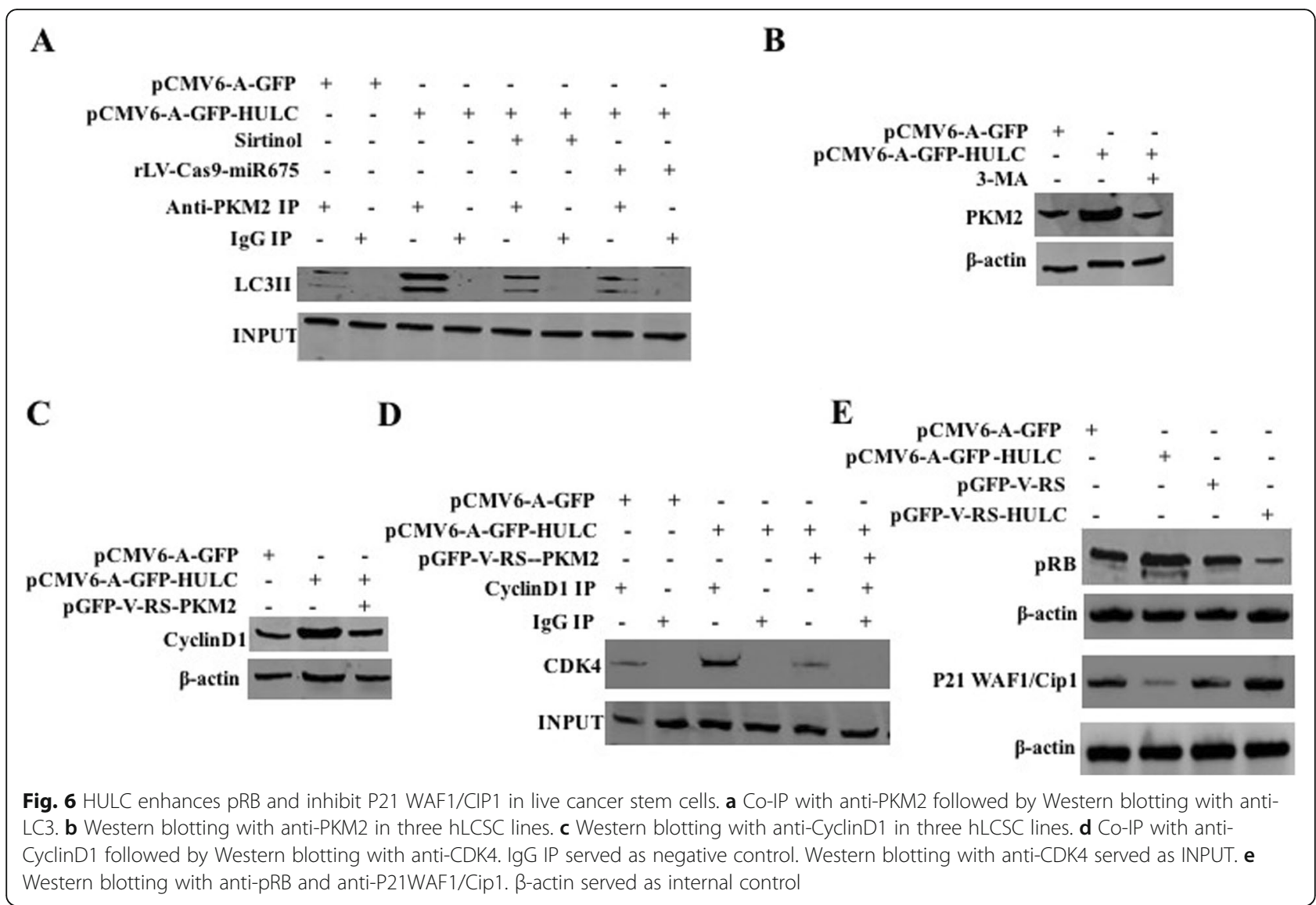

GFP group. However, the interaction between LC3II and PKM2 was not significantly altered in the pCMV6-A-GFPHULC plus Sirtinol group and the pCMV6-A-GFP-HULC plus rLV-Cas9-miR675 group compared to the pCMV6-AGFP group, respectively. Moreover, the expression of PI3K and PKM2 was significantly increased in the pCMV6-AGFP-HULC group compared to the pCMV6-A-GFP group. However, the expression of PI3K and PKM2 was not significantly altered in the pCMV6-A-GFP-HULC plus 3-methyladenine (3-MA) group compared to the pCMV6A-GFP group, respectively (Fig. 6b). Therefore, the expression of CyclinD1 was significantly increased in the pCMV6A-GFP-HULC group compared to the pCMV6-A-GFP group. However, the expression of CyclnD1 was not significantly altered in the pCMV6-A-GFP-HULC plus rLV-Cas9PKM2 group compared to the pCMV6-A-GFP group, respectively (Fig. 6c). Moreover, the interaction between CDK4 and CyclinD1 was significantly enhanced in the pCMV6-A-GFP-HULC group compared to the pCMV6-AGFP group. However, the interaction between CDK4 and CyclinD1 was significantly not altered in the pCMV6-AGFP-HULC plus pGFP-V-RS-PKM2 group compared to the pCMV6-A-GFP group (Fig. 6d). Finally, pRB was significantly increased in the pCMV6-A-GFP-HULC group compared to the pCMV6-A-GFP group and reduced in the
pGFP-V-RS-HULC group compared to the pGFP-VRS group, and P21WAF1/Cip1 was significantly decreased in the pCMV6-A-GFP-HULC group compared to the pCMV6-A-GFP group and increased in the pGFPV-RS-HULC group compared to the pGFP-V-RS group (Fig. 6e). Collectively, these observations suggest that HULC enhances CyclinD1 to increase pRB and inhibit P21 WAF1/CIP 1 via autophagy-PKM2 pathway in human liver cancer stem cells.

CyclinD1 is required for the oncogenic functions of HULC To validate whether CyclinD1 is required for the action of HULC in liver cancer stem cells, we performed the rescuedtest. As shown in Fig. 7a, HULC expression was significantly increased in the pCMV6-A-GFP-HULC group and the pCMV6-A-GFP-HULC plus pGFP-V-RS-CyclinD1 group compared with the pCMV6-A-GFP group respectively, and CyclinD1 was significantly increased in the pCMV6-A-GFPHULC group and decreased in the pCMV6-A-GFP-HULC plus rLV-Cas9-CyclinD1 group compared with the pCMV6A-GFP group respectively. Next, as shown in Fig. 7b, although the growth of LCSCs was more rapid in the pCMV6-A-GFP-HULC group than in pCMV6-A-GFP $(P<$ 0.01 ), it was not significantly altered in the pCMV6-A-GFPHULC plus pGFP-V-RS-CyclinD1 group compared to the 


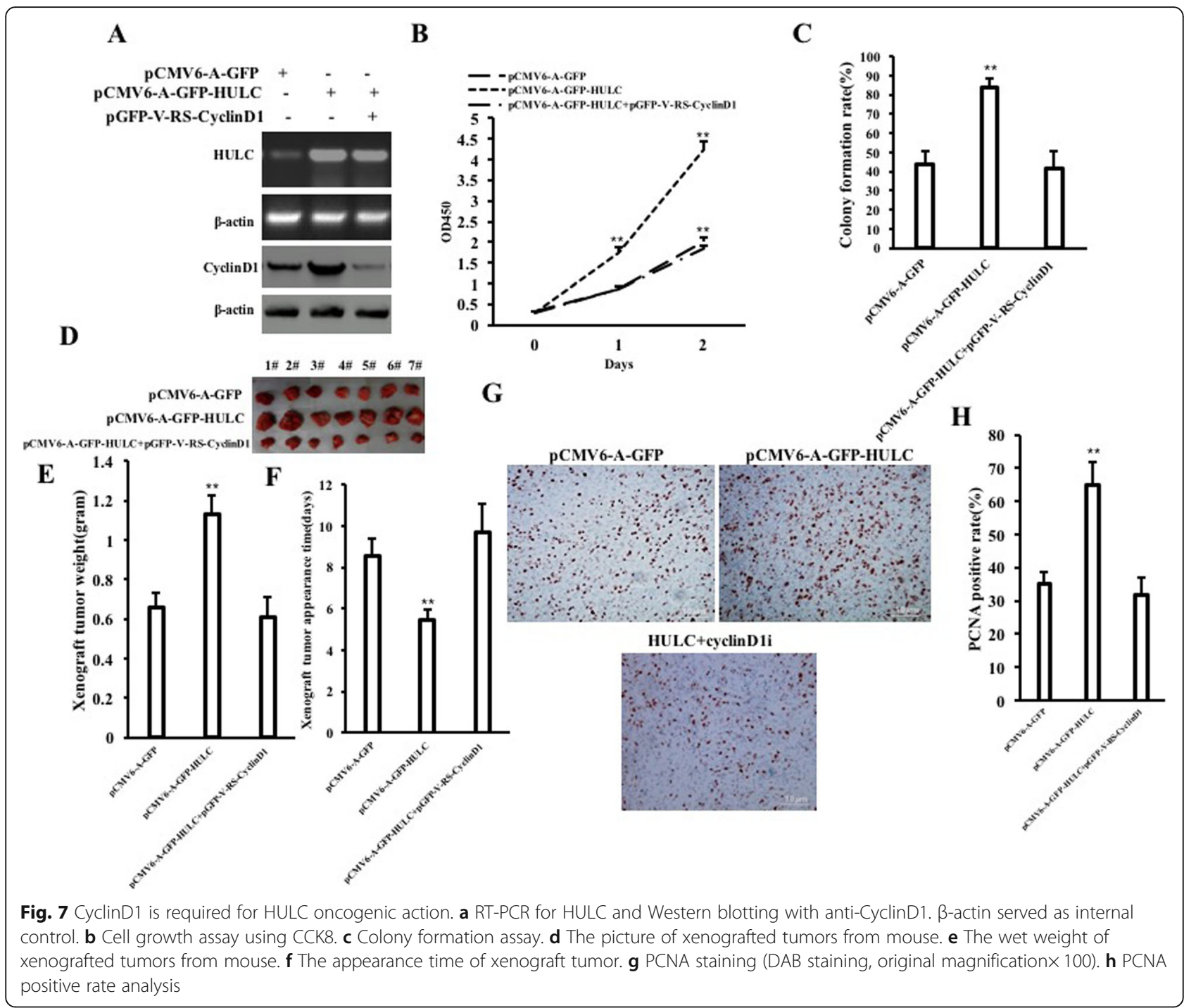

pCMV6-A-GFP control group $(P>0.05)$. As shown in Fig. $7 \mathrm{c}$, although the colony formation ability was significantly increased in the pCMV6-A-GFP-HULC group compared to the pCMV6-A-GFP group $(43.49 \pm 6.78 \%$ vs $83.86 \pm 4.28 \%, p=0.0097138<0.01$ ), it was significantly not altered in the pCMV6-A-GFP-HULC plus pGFP-V-RSCyclinD1 group compared to the pCMV6-A-GFP group $(P>0.05) \quad(43.49 \pm 6.78 \%$ vs $41.49 \pm 9.07 \%, p=0.14058>$ 0.05). As shown in Fig. $7 d$, e, although the xenograft tumor weight increased approximately twofold in the pCMV6-AGFP-HULC group compared to the pCMV6-A-GFP group $(0.657 \pm 0.069 \mathrm{~g}$ versus $1.13 \pm 0.093 \mathrm{~g}, P=0.000017<0.01)$, it was not significantly altered in the pCMV6-A-GFP-HULC plus pGFP-V-RS-CyclinD1 group compared to the pCMV6A-GFP group $(0.657 \pm 0.069 \mathrm{~g}$ versus $0.609 \pm 0.101 \mathrm{~g}, P=$ $0.14394>0.05$ ). Although the appearance time of xenograft was significantly decreased in the pCMV6-A-GFP-HULC group compared to the pCMV6-A-GFP group (8.571 \pm
0.787 days versus $5.429 \pm 0.535$ days, $P=0.00000991<0.01$ ), it was significantly not altered in the pCMV6-A-GFP-HULC plus pGFP-V-RS-CyclinD1 group compared to the pCMV6A-GFP group ( $8.571 \pm 0.787$ days versus $9.714 \pm 1.3801$ days, $p=0.086154>0.05$ ) (Fig. 7f). As shown in Fig. 7g, h, although PCNA-positive rate was significantly higher in the pCMV6-A-GFP-HULC group than in the pCMV6-A-GFP group $(35.08 \pm 3.45 \%$ versus $64.83 \pm 7.05 \%, p=0.000101<$ 0.01 ), it was not significantly altered in the pCMV6-A-GFPHULC plus pGFP-V-RS-CyclinD1 group compared to the pCMV6-A-GFP group $(35.08 \pm 3.45 \%$ versus $31.704 \pm$ $5.143 \%, p=0.1095>0.05$ ). Collectively, findings suggest that HULC accelerates progression of human liver cancer stem cells dependent on CyclinD1.

\section{Discussion}

To date, the functions and regulatory mechanism of long noncoding RNA HULC in liver cancer stem cells have 
not fully been elucidated. To our knowledge, this paper might be the first to demonstrate that HULC accelerates the growth of human liver cancer stem cells by upregulating CyclinD1 by miR675-PKM2 pathway via autophagy. In this study, we first demonstrate that HULC accelerates growth of liver cancer stem cells in vitro and in vivo. Mechanistically, HULC enhances the expression of Sirt1 dependent on miR675 and then induces the cellular autophagy through Sirt1. HULC enhances CyclinD1 and thereby increases pRB and inhibited P21 WAF1/CIP 1 via autophagy-Pyruvate Kinase M2 (PKM2) pathway in human liver cancer stem cells. Ultimately, our results demonstrate that CyclinD1 is required for the oncogenic functions of HULC in human liver cancer stem cells. These observations suggest that HULC accelerates progression of human liver cancer stem cells in vitro and in vivo dependent on CyclinD1(Fig. 8).
It is worth mentioning that our findings in this study provide novel evidence for an oncogenic active role of HULC in hLCSCs. This assertion is based on several observations: (a) HULC accelerates growth in vitro of liver cancer stem cells. (b) HULC accelerates growth in vivo of liver cancer stem cells. Several studies indicate that HULC promotes proliferation and migration [34-38]. Our present results are consistent with these reports and provide novel evidence for an active role of HULC in promoting malignant formation and growth of LCSCs. Therefore, we believe that HULC plays a key role in the development of liver cancer.

Importantly, our results suggest that HULC increases the miR675 in human liver cancer stem cells. This assertion is based on several observations: (a) excessive HULC increases and HULC knockdown decreased the binding of METTL3 (a RNA methyltransferase) to pri-miR675. (b) pri-miR675,

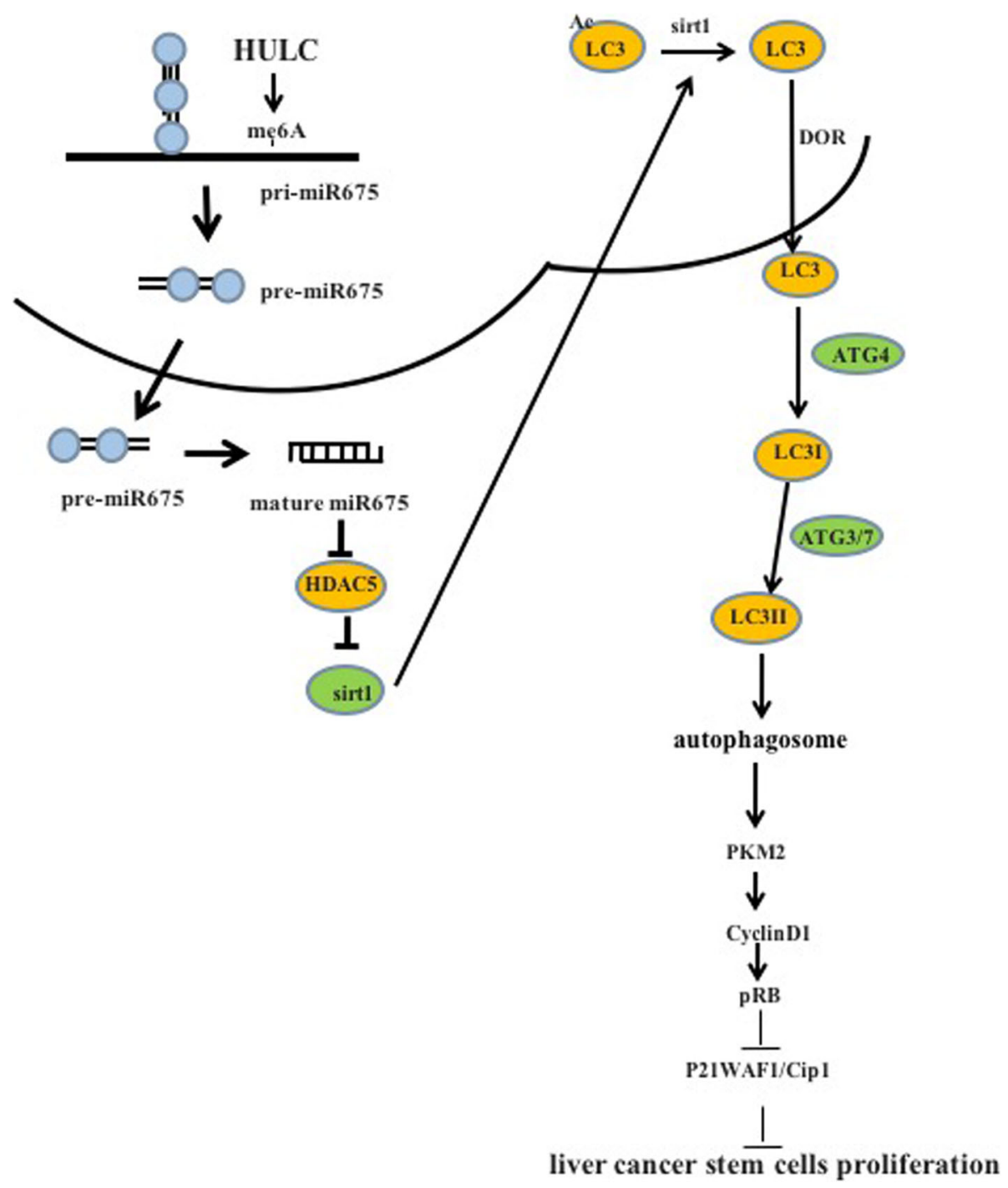

Fig. 8 Schematic illustration of the role of HULC in hLCSCS 
pre-miR675, and mature miR-R675 were significantly increased in HULC overexpressing hLCSCs. (c) The premiR675, pre-miR675, and mature mi-R675 were not significantly altered in the pCMV6-A-GFP-HULC+pGFP-V-RSMETTL3 group. (d) HULC enhances the expression and maturity of miR675 dependent on METTL3 in human liver cancer liver cells. A study showed that miR-675 promoted cancer cell growth [39]. Moreover, miR675 blocks DNA mismatch repair in cancer [40]. Our present results are consistent with these reports and provide novel evidence for an active role of HULC in promoting malignant growth of LCSCs dependent on miR-675. Thus, it suggests that miR675 plays an important role for HULC oncogenic actions.

Evidentially, our findings in this study provide novel evidence that HULC promotes the expression of Sirt1 dependent on miR675. This assertion is based on several observations: (a) miR675 targets HDAC5 mRNA3'-UTR via eight-seed sequence. (b) DHAC5 was significantly reduced in the rLV-miR675 group. (c) The loading of DHAC5 on the Sirt1 promoter region was significantly reduced in the pCMV6-A-GFP-HULC group. (d) The luciferase activity of Sirt1 promoter was significantly increased in the pCMV6-AGFP-HULC group. (e) The expression of Sirt1 was significantly increased in the pCMV6-A-GFP-HULC group. (f) HULC enhances the expression of Sirt1 dependent on miR675-HDAC5. A study shows that SIRT1 enhances the metabolic flexibility [41]. Moreover, SIRT1 promotes glucose transporting [42] and inhibits apoptosis of cancer cells [43]. Our present results are consistent with these reports. Therefore, miR675-HDAC5-Sirt1 axis regulates the oncogenic functions of HULC. However, it should be explored further.

Notably, our results suggest that HULC increases the autophagy through Sirt1. This evidence is based on results from nine parallel sets of experiments: (a) the interaction between Sirt1 and LC3 was significantly increased in the pCMV6-A-GFP-HULC group. (b) The Ac-LC3 was significantly decreased in the pCMV6-A-GFP-HULC group. (c) The interaction between LC3 and DOR was significantly increased in the pCMV6-A-GFP-HULC group. (d) The interaction between LC3 and ATG4 was significantly increased in the pCMV6-A-GFP-HULC group. (e) The interaction between LC3 and ATG3 was significantly increased in the pCMV6-A-GFP-HULC group. (f) The interaction between ATG3 and ATG7 was significantly increased in the pCMV6-A-GFP-HULC group. (g) The activated LC3II was significantly enhanced in the pCMV6-A-GFP-HULC group. (h) The autophagy was significantly enhanced in the pCMV6-A-GFP-HULC group. (i) HULC increases the autophagy dependent on Sirt1.

Therefore, HULC enhances the expression of Sirt1 dependent on miR675 and therefore increases the autophagy through Sirt1.
Strikingly, HULC enhances CyclinD1 to increase pRB and inhibit P21 WAF1/CIP 1 via autophagy-PKM2 pathway. This evidence is based on results from three parallel sets of experiments: (a) the interaction between LC3II and PKM2 was significantly enhanced in the pCMV6-A-GFP-HULC group. (b) The expression of PI3K and PKM2 was significantly increased in the pCMV6-A-GFP-HULC group. However, the expression of PI3K and PKM2 was not significantly altered in the pCMV6-A-GFP-HULC plus 3-methyladenine (3-MA) group. (c) The expression of CyclinD1 was significantly increased in the pCMV6-A-GFP-HULC group. (d) The interaction between CDK4 and CyclinD1 was significantly enhanced in the pCMV6-A-GFP-HULC group. (e) pRB was significantly increased in the pCMV6-A-GFPHULC group and reduced in the pGFP-V-RS-HULC group. (f) HULC enhances CyclinD1 to increase pRB and inhibit P21 WAF1/CIP1 via autophagy-PKM2 pathway in human liver cancer stem cells. A study shows that autophagy impairs endothelial function [44] and ubiquitination of MAP 1LC3B is associated with autophagy [45]. Interestingly, TLR2 enhances autophagy [46]. Importantly, PKM2 promotes cell survival [47] and regulates STAT3 [48]. In addition, miR-625-5p/PKM2 regulates glycolysis state [49]. Moreover, pRb-E2F pathway induced growth of cancer cells [50]. In particular, microRNA-16-5p modulates Cyclin D1/ E1-pRb-E2F1 pathway in cancer cells [51]. Moreover, HPV-16 E7 regulates phospholipase D activity in a pRBdependent manner [52]. A study shows that lincRNA-p21 acts as a tumor suppressor [53] and SPSB1 destabilizes p21WAF1/Cip1 [54]. Our present results are consistent with these reports and provide novel evidence for oncogenic role of HULC in promoting malignant growth of LCSCs via CyclinD1-pRB-P21WAF1/CIP 1 via autophagyPKM2 pathway. Therefore, HULC oncogenic action is associated with PKM2, CyclinD1, pRB, P21 WAF1/CIP1, and cellular autophagy in human liver cancer stem cells.

Another significant finding is that CyclinD1 is required for the oncogenic functions of HULC. This evidence is based on results from five parallel sets of experiments: (a) although the growth of LCSCs was more rapid in the pCMV6-A-GFP-HULC group, it was not significantly altered in the pCMV6-A-GFP-HULC plus pGFP-V-RSCyclinD1 group. (b) Although the colony formation ability of LCSCs was significantly increased in the pCMV6A-GFP-HULC group, it was not significantly altered in the pCMV6-A-GFP-HULC plus pGFP-V-RS-CyclinD1 group. (c) Although the xenograft tumor weight was increased in the pCMV6-A-GFP-HULC group, it was not significantly altered in the pCMV6-A-GFP-HULC plus pGFP-V-RS-CyclinD1 group. (d) Although the appearance time of xenograft was significantly decreased in the pCMV6-A-GFP-HULC group, it was not significantly altered in the pCMV6-A-GFP-HULC plus pGFP-V-RSCyclinD1 group. (e) HULC accelerates progression of 
human liver cancer stem cells dependent on CyclinD1. A study indicated that CyclinD1 polymorphism modified susceptibility oncogene [55]. In particular, miR-760 suppresses cancer growth by targeting cyclinD1 [56]. Furthermore, Cyclin D1 integrates histone methylation [57]. Our present results are consistent with these reports and provide novel evidence for oncogenic role of HULC in promoting malignant growth of LCSCs through CyclinD1.

In summary, HULC promotes growth of liver cancer stem cells in vitro and in vivo. Mechanistically, HULC enhances the expression of Sirt1 dependent on miR675 and then induces the cellular autophagy through Sirt1. HULC enhances CyclinD1 and thereby increases PRB and inhibited P21 WAF1/CIP 1 via autophagy-PKM2 pathway in human liver cancer stem cells. Ultimately, our results demonstrate that CyclinD1 is required for the oncogenic functions of HULC in human liver cancer stem cells. These observations provide important basic information for finding effective liver cancer therapeutic targets. Therefore, governing HULC expression will be crucial for the identification of novel liver cancer therapeutic strategies. We will further study the exact mechanism of HULC in the development of liver cancer and its clinical application.

\section{Conclusions}

Long noncoding RNA HULC accelerates growth of liver cancer stem cells by enhancing the expression of Sirt1 dependent on miR675 and then inducing the cellular autophagy to increase CyclinD1 and pRB in human liver cancer stem cells. In particular, CyclinD1 is required for the oncogenic functions of HULC in human liver cancer stem cells. These observations provide important basic information for finding effective liver cancer therapeutic targets. Therefore, governing HULC expression will be crucial for the identification of novel liver cancer therapeutic strategies.

\section{Supplementary information}

Supplementary information accompanies this paper at https://doi.org/10. 1186/s13287-019-1528-y.

\footnotetext{
Additional file 1: Figure S1. The isolation and identification of human liver cancer stem cell. A. The transcriptional ability of CD133, CD44, CD24, and Epcam was analyzed by reverse transcription polymerase chain reaction, and $\beta$-actin was used as an internal reference gene. B. Western blotting analysis using anti-CD133, anti-CD44, anti-CD24, anti-EpCAM, and $\beta$-actin as an internal reference gene. Figure S2. A. The assay of sphere formation rate in hLCSCs and non- hLCSCs. B. tumorigenesis test in vivo in hLCSCs and non- hLCSCs. Figure S3. A. Northern blotting with BiotinmiR675 probe in pCMV6-A-GFP group, pCMV6-A-GFP-HULC group and pCMV6-A-GFP-HULC+pGFP-V-RS-METTL3 group. U6 served as the internal control. B. Real-time RT-PCR with miR675 primers in pCMV6-A-GFP group, pCMV6-A-GFP-HULC group and pCMV6-A-GFP-HULC+pGFP-V-RS-METTL3 group. U6 served as an internal control. Figure S4. The analysis of HDAC5 3'UTR (mutant) luciferase reporter activity in rLV-miR675 group and $\mathrm{rLV}$ control group.
}

\section{Abbreviations}

HULC: Highly upregulated in liver cancer; RIP : RNA immunoprecipitation; CHIP : Chromatin immunoprecipitation; 3'-UTR : 3' untranslational region; LCSC : Liver cancer stem cell; IGF2BP1: IGF2 mRNA-binding protein 1; SIRT1 : Sirtuin 1; BMP: Bone morphogenetic protein; HSPC : Hematopoietic stem and progenitor cell; EEF1E1: Eukaryotic translation elongation factor 1 epsilon-1; BNIP3 : BCL2 Interacting Protein 3; PKM2: Pyruvate Kinase M2; MALAT1 : Metastasis Associated Lung Adenocarcinoma Transcript 1; KDM4B : Lysine-specific demethylase 4B; GLUT1 : Glucose transporter protein 1; HPV16 : Human papillomavirus 16; HDAC5: Histone deacetylase 5; 3-MA: 3Methyladenine

\section{Authors' contributions}

DL conceived the study and participated in the study design, performance, coordination, and manuscript writing. CW, XXJ, XNL, STS, QYM, LYW, YNL, $X R X, H P, X G$, and TML performed the research. All authors have read and approved the final manuscript.

\section{Funding}

This study was supported by grants from National Natural Science Foundation of China (NCSF No.81572773) and National Natural Science Foundation of China (NCSF No.81773158) and by grants from Science and Technology Commission of Shanghai Municipality Basic Research Field Project (19JC1415200).

\section{Availability of data and materials}

Not applicable.

\section{Ethics approval and consent to participate}

All methods were carried out in "accordance" with the approved guidelines. All experimental protocols "were approved by" Tongji University Institutional Committee. The study was reviewed and approved by the China National Institutional Animal Care and Use Committee.

\section{Consent for publication}

Not applicable.

\section{Competing interests}

The authors declare that they have no competing interests.

Received: 20 June 2019 Revised: 30 October 2019 Accepted: 10 December 2019 Published online: 03 January 2020

\section{References}

1. Panzitt K, Tschernatsch MM, Guelly C, Moustafa T, Stradner M, Strohmaier HM, Buck CR, Denk H, Schroeder R, Trauner M, Zatloukal K. Characterization of HULC, a novel gene with striking up-regulation in hepatocellular carcinoma, as noncoding RNA. Gastroenterology. 2007;132(1):330-42.

2. Yu X, Zheng H, Chan MT, WU WK. HULC: an oncogenic long non-coding RNA in human cancer. J Cell Mol Med. 2017;21(2):410-7.

3. Lin Z, Lu Y, Meng Q, Wang C, Li X, Yang Y, Xin X, Zheng Q, Xu J, Gui X, Li T, Pu H, Xiong W, Li J, Jia S, Lu D. miR372 promotes progression of liver cancer cells by upregulating erbB-2 through enhancement of YB-1. Mol Ther Nucleic Acids. 2018;11:494-50.

4. Zhao L, Man Y, Liu S. Long non-coding RNA HULC promotes UVB-induced injury by up-regulation of BNIP3 in keratinocytes. Biomed Pharmacother. 2018;104:672-8.

5. Wang BG, Lv Z, Ding HX, Fang XX, Wen J, Xu Q, Yuan Y. The association of IncRNA-HULC polymorphisms with hepatocellular cancer risk and prognosis. Gene. 2018;670:148-54.

6. Zheng P, Li H, Xu P, Wang X, Shi Z, Han Q, Li Z. High IncRNA HULC expression is associated with poor prognosis and promotes tumor progression by regulating epithelial-mesenchymal transition in prostate cancer. Arch Med Sci. 2018;14(3):679-86.

7. Chen C, Wang K, Wang Q, Wang X. LnCRNA HULC mediates radioresistance via autophagy in prostate cancer cells. Braz J Med Biol Res. 2018;51(6):e7080

8. Li Y, Ge C, Feng G, Xiao H, Dong J, Zhu C, Jiang M, Cui M, Fan S. Low dose irradiation facilitates hepatocellular carcinoma genesis involving HULC. Mol Carcinog. 2018 Jul;57(7):926-35. 
9. Li Y, Shan Z, Yang B, Yang D, Men C, Cui Y, Wu J. LncRNA HULC promotes epithelial and smooth-muscle-like differentiation of adipose-derived stem cells by upregulation of BMP9. Pharmazie. 2018;73(1):49-55.

10. Chen S, Wu DD, Sang XB, Wang LL, Zong ZH, Sun KX, Liu BL, Zhao Y. The IncRNA HULC functions as an oncogene by targeting ATG7 and ITGB1 in epithelial ovarian carcinoma. Cell Death Dis. 2017 Oct 12;8(10):e3118.

11. Wang J, Ma W, Liu Y. Long non-coding RNA HULC promotes bladder cancer cells proliferation but inhibits apoptosis via regulation of ZIC2 and PI3KJAKT signaling pathway. Cancer Biomark. 2017;20(4):425-34.

12. Kong D, Wang Y. Knockdown of IncRNA HULC inhibits proliferation, migration, invasion, and promotes apoptosis by sponging miR-122 in osteosarcoma.J Cell Biochem 2018;119(1):1050-1061.

13. Xiong H, Li B, He J, Zeng Y, Zhang Y, He F. IncRNA HULC promotes the growth of hepatocellular carcinoma cells via stabilizing COX-2 protein. Biochem Biophys Res Commun. 2017:490(3):693-9.

14. Xiong $\mathrm{H}, \mathrm{Ni}$ Z, He J, Jiang S, Li X, He J, Gong W, Zheng L, Chen S, Li B, Zhang N, Lyu X, Huang G, Chen B, Zhang Y, He F. LncRNA HULC triggers autophagy via stabilizing Sirt1 and attenuates the chemosensitivity of HCC cells. Oncogene. 2017;36(25):3528-40.

15. Wu M, Lin Z, Li X, Xin X, An J, Zheng Q, Yang Y, Lu D. HULC cooperates with MALAT1 to aggravate liver cancer stem cells growth through telomere repeat-binding factor 2. Sci Rep. 2016;6:36045.

16. Gui $X, \mathrm{Li} \mathrm{H}, \mathrm{Li}$ T, Pu H, Lu D. Long noncoding RNA CUDR regulates HULC and $\beta$-catenin to govern human liver stem cell malignant differentiation. Mol Ther. 2015;23(12):1843-53.

17. Hämmerle M, Gutschner T, Uckelmann H, Ozgur S, Fiskin E, Gross M, Skawran B, Geffers R, Longerich T, Breuhahn K, Schirmacher P, Stoecklin G, Diederichs S. Posttranscriptional destabilization of the liver-specific long noncoding RNA HULC by the IGF2 mRNA-binding protein 1 (IGF2BP1) Hepatology. 2013;58(5):1703-12.

18. Liu L, Tian YC, Mao G, Zhang YG, Han L. Moreover, miR-675 is frequently overexpressed in gastric cancer and enhances cell proliferation and invasion via targeting a potent anti-tumor gene PITX1. Cell Signal. 2019;62:109352.

19. Zheng ZH, Wu DM, Fan SH, Zhang ZF, Chen GQ, Lu J. In particular, upregulation of miR-675-5p induced by IncRNA H19 was associated with tumor progression and development by targeting tumor suppressor p53 in non-small cell lung cancer. J Cell Biochem. 2019 Jun 20. https://doi.org/10. 1002/jcb.29182.

20. Wang L, Jiang P, He Y, Hu H, Guo Y, Liu X, Qiu H, Ma Q, Ouyang F. A nove mechanism of Smads/miR-675/TGFBR1 axis modulating the proliferation and remodeling of mouse cardiac fibroblasts. J Cell Physiol. 2019;234(11): 20275-85.

21. Morgoulis D, Berenstein P, Cazacu S, Kazimirsky G, Dori A, Barnea ER, Brodie C. sPIF promotes myoblast differentiation and utrophin expression while inhibiting fibrosis in Duchenne muscular dystrophy via the H19/miR-675/let7 and miR-21 pathways. Cell Death Dis. 2019;10(2):82.

22. Pan JX, Chen TN, Ma K, Wang S, Yang CY, Cui GY. A negative feedback loop of H19/miR-675/NDR mediates therapeutic effect of cucurmin in the treatment of glioma. J Cell Physiol. 2019. https://doi.org/10.1002/jcp.29127.

23. Park SM, Kim J, Hong CM, Shin DH, Kim JY, Park DY, Sohn DH, Kim YH Kwon SM, Kim JH, Bae SS, Kim K, Kim CD, Kang CD, Lee D. SIRT1 is dispensable for maturation of hematopoietic stem cell in the bone marrow niche. Exp Ther Med. 2019;18(3):2341-5.

24. Shi Y, Yang Z, Zhang T, Shen L, Li Y, Ding S. SIRT1-targeted miR-543 autophagy inhibition and epithelial-mesenchymal transition promotion in Helicobacter pylori CagA-associated gastric cancer. Cell Death Dis. 2019; 10(9):625.

25. Qi H, Wang $H$, Pang D. miR-448 promotes progression of non-small-cell lung cancer via targeting SIRT1. Exp Ther Med. 2019;18(3):1907-13.

26. Wei $Y$, Huang $C$, Wu H, Huang J. Estrogen receptor beta (ER $\beta$ ) mediatedCyclinD1 degradation via autophagy plays an anti-proliferation role in colon cells. Int J Biol Sci. 2019;15(5):942-52.

27. Dreyer JH, Hauck F, Barros MHM, Niedobitek G. pRb and CyclinD1 complement p16 as immunohistochemical surrogate markers of HPV infection in head and neck cancer. Appl Immunohistochem Mol Morphol. 2017:25(5):366-73.

28. Ru Y, Chen XJ, Zhao ZW, Zhang PF, Feng SH, Gao Q, Gao SG, Feng XS Interestingly,p16-positive/HPV DNA-PCR-negative cases show heterogeneous expression of $\mathrm{pRb}$ and CyclinD1, including high $\mathrm{pRb}$ or high combined $\mathrm{pRb} /$ CyclinD1 scores suggesting that at least some of these cases are truly HPV negative. Oncotarget. 2017;8(43):73860-70.
29. Ravanan P, Srikumar IF, Talwar P. Autophagy: The spotlight for cellular stress responses. Life Sci. 2017;88:53-67.

30. Wang QX, Lv L, Ye DR, Sun YH, Pan XX, Bhandari A, Zhang XH, Wang OC, Liu HG. Downregulation of CD44v6 enhances chemosensitivity by promoting apoptosis and inhibiting autophagy in colorectal cancer HT29 cells. Ann Clin Lab Sci. 2019;49(4):481-7.

31. Su Y, Yao S, Zhao S, Li J, Li H. LncRNA CCAT1 functions as apoptosis inhibitor in podocytes via autophagy inhibition. J Cell Biochem. 2019 Aug 29. https://doi.org/10.1002/jcb.29307.

32. Sha J, Han Q, Chi C, Zhu Y, Pan J, Dong B, Huang Y, Xia W, Xue W. Upregulated KDM4B promotes prostate cancer cell proliferation by activating autophagy. J Cell Physiol. 2019 Aug 29. https://doi.org/10.1002/jcp.29117.

33. Smith LD, Minton AR, Blunt MD, Karydis LI, Dutton DA, Rogers-Broadway KR, Dobson R, Liu R, Norster F, Hogg E, Ashton-Key M, Strefford JC, Jia L, Efremov DG, Helgason GV, Johnson PWM, Stevenson FK, Forconi F, Cragg MS, Tumbarello DA, Packham G, Steele AJ. BCR signaling contributes to autophagy regulation in chronic lymphocytic leukemia. Leukemia. 2019 Aug 28. https://doi.org/10.1038/s41375-019-0557-y.

34. Su W, Tang J, Wang Y, Sun S, Shen Y, Yang H. Long non-coding RNA highly up-regulated in liver cancer promotes epithelial-to-mesenchymal transition process in oral squamous cell carcinoma. J Cell Mol Med. 2019 Jan 24 https://doi.org/10.1111/jcmm.14160

35. Feng $H$, Wei B, Zhang Y. Long non-coding RNA HULC promotes proliferation, migration and invasion of pancreatic cancer cells by downregulating microRNA-15a. Int J Biol Macromol. 2018;126:891-8.

36. Abbastabar M, Sarfi M, Golestani A, Khalili E. IncRNA involvement in hepatocellular carcinoma metastasis and prognosis. EXCLI J. 2018;17:900-13.

37. Xin X, Wu M, Meng Q, Wang C, Lu Y, Yang Y, Li X, Zheng Q, Pu H, Gui X, Li T, Li J, Jia S, Lu D. Long noncoding RNA HULC accelerates liver cancer by inhibiting PTEN via autophagy cooperation to miR15a. Mol Cancer. 2018; 17(1):94.

38. Jiang XR, Guo N, Li XQ, Yang HY, Wang K, Zhang CL, Li GS, Li GD. Long non-coding RNA HULC promotes proliferation and osteogenic differentiation of bone mesenchymal stem cells via down-regulation of miR-195. Eur Rev Med Pharmacol Sci. 2018;22(10):2954-65.

39. Yang $X$, Lou $Y$, Wang M, Liu C, Liu Y, Huang W. miR-675 promotes colorectal cancer cell growth dependent on tumor suppressor DMTF1. Mol Med Rep. 2019;19(3):1481-90.

40. Lu Y, Song S, Jiang X, Meng Q, Wang C, Li X, Yang Y, Xin X, Zheng Q, Wang L, Pu H, Gui X, Li T, Lu D. miR675 accelerates malignant transformation of mesenchymal stem cells by blocking DNA mismatch repair. Mol Ther Nucleic Acids. 2019;14:171-83.

41. Wei Z, Jia J, Heng G, Xu H, Shan J, Wang G, Liu C, Xia J, Zhou H, Wu M, Yang Z, Wang M, Xiong Z, Huang H, Liu L, Qian C. SIRT1/MRPS5 axis enhances the metabolic flexibility of liver cancer stem cells. Hepatology. 2019 Mar 22. https://doi.org/10.1002/hep.30622.

42. Chen J, Cao L, Li Z, Li Y. SIRT1 promotes GLUT1 expression and bladder cancer progression via regulation of glucose uptake. Hum Cell. 2019 Mar;13. https://doi.org/10.1007/s13577-019-00237-5.

43. Ye G, Kan S, Chen J, Lu X. Puerarin in inducing apoptosis of bladder cancer cells through inhibiting SIRT1/p53 pathway. Oncol Lett. 2019;17(1):195-200.

44. Chen P, Liu H, Xiang H, Zhou J, Zeng Z, Chen R, Zhao S, Xiao J, Shu Z, Chen $\mathrm{S}$, Lu H. Palmitic acid-induced autophagy increases reactive oxygen species via the $\mathrm{Ca}_{2+} / \mathrm{PKCa} / \mathrm{NOX} 4$ pathway and impairs endothelial function in human umbilical vein endothelial cells. Exp Ther Med. 2019;17(4):2425-32.

45. Kang HM, Noh KH, Chang TK, Park D, Cho HS, Lim JH, Jung CR. Ubiquitination of MAP1LC3B by pVHL is associated with autophagy and cell death in renal cell carcinoma. Cell Death Dis. 2019;10(4):279.

46. Li C, Ma L, Liu Y, Li Z, Wang Q, Chen Z, Geng X, Han X, Sun J, Li Z. TLR2 promotes development and progression of human glioma via enhancing autophagy. Gene. 2019.

47. Qi H, Ning X, Yu C, Ji X, Jin Y, McNutt MA, Yin Y. Succinylation-dependent mitochondrial translocation of PKM2 promotes cell survival in response to nutritional stress. Cell Death Dis. 2019;10(3):170. https://doi.org/10.1038/ s41419-018-1271-9.

48. Ma R, Liu Q, Zheng S, Liu T, Tan D, Lu X. PKM2-regulated STAT3 promotes esophageal squamous cell carcinoma progression via TGF- $\beta 1$-induced EMT. I Cell Biochem. 2019. https://doi.org/10.1002/jcb.28434.

49. Zhang $H$, Feng C, Zhang M, Zeng A, Si L, Yu N, Bai M. miR-625-5p/ PKM2 negatively regulates melanoma glycolysis state. J Cell Biochem. 2019;120(3):2964-72. 
50. Henry $\mathrm{D}$, Brumaire $\mathrm{S}, \mathrm{Hu} \mathrm{X}$. Involvement of pRb-E2F pathway in green tea extract-induced growth inhibition of human myeloid leukemia cells. Leuk Res. 2019;77:34-41.

51. Wang F, Mao A, Tang J, Zhang Q, Yan J, Wang Y, Di C, Gan L, Sun C, Zhang H. microRNA-16-5p enhances radiosensitivity through modulating Cyclin D1/E1-pRb-E2F1 pathway in prostate cancer cells.J Cell Physiol. 2018. doi: https://doi.org/10.1002/jcp.27989

52. Rabachini T, Boccardo E, Andrade R, Perez KR, Nonogaki S, Cuccovia IM, Villa LL. HPV-16 E7 expression up-regulates phospholipase D activity and promotes rapamycin resistance in a pRB-dependent manner. BMC Cancer. 2018;18(1):485.

53. Jin S, Yang X, Li J, Yang W, Ma H, Zhang Z. p53-targeted lincRNA-p21 acts as a tumor suppressor by inhibiting JAK2/STAT3 signaling pathways in head and neck squamous cell carcinoma. Mol Cancer. 2019;18(1):38.

54. Kim HJ, Kim MK, Bae MK, Sung HY, Ahn JH, Kim YH, Kim SC, Ju W. SPSB enhances ovarian cancer cell survival by destabilizing p21. Biochem Biophys Res Commun. 2019;510(3):364-9.

55. Pandey A, Bahl C, Sharma S, Singh N, Behera D. Functional role of CyclinD1 polymorphism (G870A) in modifying susceptibility and overall survival of North Indian lung cancer patients. Tumori. 2018;104(3):179-87.

56. Cao L, Liu Y, Wang D, Huang L, Li F, Liu J, Zhang C, Shen Z, Gao Q, Yuan W, Zhang Y. MiR-760 suppresses human colorectal cancer growth by targeting BATF3/AP-1/cyclinD1 signaling. J Exp Clin Cancer Res. 2018;37(1):83.

57. Li Z, Jiao X, Di Sante G, Ertel A, Casimiro MC, Wang M, Katiyar S, Ju X, Klopfenstein DV, Tozeren A, Dampier W, Chepelev I, Jeltsch A, Pestell RG. Cyclin D1 integrates G9a-mediated histone methylation. Oncogene. 2019. https://doi.org/10.1038/s41388-019-0723-8.

\section{Publisher's Note}

Springer Nature remains neutral with regard to jurisdictional claims in published maps and institutional affiliations.

Ready to submit your research? Choose BMC and benefit from:

- fast, convenient online submission

- thorough peer review by experienced researchers in your field

- rapid publication on acceptance

- support for research data, including large and complex data types

- gold Open Access which fosters wider collaboration and increased citations

- maximum visibility for your research: over $100 \mathrm{M}$ website views per year

At $\mathrm{BMC}$, research is always in progress.

Learn more biomedcentral.com/submissions 